\title{
ОЦЕНКА ТЕХНОЛОГИЧЕСКОЙ И ЭКОНОМИЧЕСКОЙ ЭФФЕКТИВНОСТИ ГИДРАВЛИЧЕСКОГО РАЗРЫВА ПЛАСТА С ИСПОЛЬЗОВАНИЕМ ГЕОЛОГО-ГИДРОДИНАМИЧЕСКОЙ МОДЕЛИ, УЧИТЫВАЮЩАЯ ОСОБЕННОСТИ СТРОЕНИЯ КАРБОНАТНЫХ КОЛЛЕКТОРОВ
}

\author{
Менгалиев Александр Генрихович1, \\ mengaliev.alexandr@gmail.com
}

\author{
Мартюшев Дмитрий Александрович², \\ martyushevd@inbox.ru \\ 1 Филиал ООО «ЛУКОЙЛ-Инжиниринг» «ПермНИПИнефть» в г. Перми, \\ Россия, 614000, г. Пермь, ул. Советской Армии, 29. \\ 2 Пермский национальный исследовательский политехнический университет, \\ Россия, 614990, г. Пермь, пр. Комсомольский, 29.
}

\begin{abstract}
Актуальность работы. В настоящее время одним из эфффективных методов интенсифрикации добычи нефрти на территории Пермского края и во всем мире является гидравлический разрыв пласта. Проведенные мероприятия гидравлического разрыва пласта часто сопровождаются увеличением обводненности добываемой продукции в виду того, что в процессе проведения мероприятия трещина гидравлического разрыва пласта распространилась в вертикальном направлении и тем самым увеличила каналы, соединяющие водонасыщенную и нефтяную зоны. Данного негативного последствия вполне можно было избежать, если бы перед проведением работ на скважине данное мероприятие было смоделировано на геологогидродинамической модели, которая учитывает особенности геологического строения данной залежи. Знание особенностей строения карбонатных залежей позволяет значительно уточнить и улучшить действующие геолого-гидродинамические модели. Соответственно использование геолого-гидродинамической модели, которые учитывают особенности строения карбонатных объектов, позволит подобрать рациональную систему разработки и обеспечить оптимальные геологотехнологические мероприятия и как следствие приведет к снижению экономических рисков.

Цель: оценка технологической и экономической эффрективности гидравлического разрыва пласта с использованием гидродинамической модели, которая учитывает геологические особенности строения карбонатной залежи.

объект: фраменская карбонатная залежь Гагаринского месторождения.

Meтоды: использование гидродинамического симулятора Tempest версии 8.3.1 компании Roxar.

Результаты. Полученные в ходе моделирования технологии гидравлического разрыва пласта на шести скважинахкандидатах результаты показали, что использование стандартной модели (применяется на сегодняшний момент) по сравнению с модифицированной геолого-гидродинамической моделью (учитывает анизотропию проницаемости и трещиноватость) приводит к завышению одних технологических показателей (прирост дебита нефтти и накопленная добьла нефрти) и к занижению других (обводненность добываемой продукции), что соответственно в последующем может привести к искажению картины экономической составляющей мероприятий. Проведенный экономический анализ позволил установить, что чистый дисконтированный доход, рассчитанный на модифицированной геолого-гидродинамической модели, оказались в 3,7 раз меньше по сравнению со стандартной, что является несомненно важными показателями при подборе скважинкандидатов для планирования геолого-технологических мероприятий.
\end{abstract}

\section{Ключевые слова:}

Вертикальная и горизонтальная проницаемости, трещиноватость, увеличение обводненности, прирост дебита нефоти, экономическая целесообразность, дополнительная добыча нефрти.

\section{Введение}

В последнее время доля нефтяных активов Пермского края, которые относятся к карбонатных объектам, заметно возросла. Данные залежи обычно представлены различными типами пустотности - поровая матрица, трещины и каверны, значительной фильтрационно-емкостной неоднородности (различие, в некоторых случаях значительное, между вертикальной и горизонтальной проницаемостью), все это в различной мере влияет на производительность добывающих скважин, коэффициент извлечения нефти, а также на эффективность проводимых геолого-технологических мероприятий (ГТМ). Знание описанных особенностей строения карбонатных залежей позволяет значительно уточнить и улучшить действующие геологогидродинамические модели (ГГДМ). Соответственно использование ГГДМ, которые учитывают особенно- сти строения карбонатных объектов, позволит подобрать рациональную систему разработки и обеспечить оптимальные ГТМ, и как следствие приведет к увеличению экономической составляющей проектов.

На сегодняшний момент при создании ГГДМ карбонатных объектов нефтяных месторождений Пермского края зачастую не учитывают описанные выше особенности их строения, особенно стоить отметить, что зачастую вертикальную проницаемость принимают равной нулю, хотя это далеко не так и об этом говорится в работах $[1,2]$. Вертикальная проницаемость в карбонатных коллекторах играет особую роль в условиях активной роли пластовых вод [3-7]. Конус подошвенной воды часто образуется при нерациональной выработке запасов и необоснованных технологических режимах скважин. В карбонатных коллекторах, имеющих вертикальные трещины, процесс 
конусообразования проявляется намного интенсивнее, чем, например, в терригенных коллекторах, так как именно они простираются далеко вниз и образуют пути для создания конусов воды.

Подводя итог вышесказанному, использование вертикальной проницаемости в ГГДМ является актуальной задачей, позволяющей повысить качество и достоверность прогнозирования процессов добычи и планируемых ГТМ. В Пермском крае около $40 \%$ проводимых ГТМ не достигают своего планируемого эффекта, хотя предварительные расчеты на ГГДМ, которые не учитывают особенности геологического строения, показали и технологическую, и экономическую эффективность их реализации $[8,9]$.

В настоящее время одним из часто применяемых и эффективных методов интенсификации добычи нефти на территории Пермского края и во всем мире является гидравлический разрыв пласта (ГРП), однако именно с этим методом интенсификации добычи нефти связан риск со значительным увеличением обводненности $[9,10]$. На территории Пермского края применяются самые разнообразные технологии проведения ГРП, такие как классический проппантный в терригенных коллекторах, кислотный - в карбонатных, кислотный ГРП с закреплением трещин проппантом, азотно-пенный ГРП и др. [10-19]. Проведенные мероприятия ГРП часто сопровождаются увеличением обводненности добываемой продукции в виду того, что в процессе проведения мероприятия трещина ГРП распространилась в вертикальном направле- нии и тем самым увеличила каналы, соединяющие водонасыщенную и нефтяную зоны [20-28]. Данное негативное последствие было отмечено после проведения кислотного гидроразрыва пласта (КГРП) на скважине 412 Гагаринского месторождения (объект Фм). Показатели эксплуатации и результаты обработки гидродинамических исследований скважин (ГДИ) представлены на рис. 1 и в табл. 1. Проведение КГРП в мае 2011 г. не привело к увеличению дебита нефти, зато значительно увеличилась обводненность добываемой продукции (с 1 до 99 \%). Интерпретация данных ГДИ позволила установить, что горизонтальная проницаемость увеличилась незначительно (с 10,1 до 55,7 мД) по сравнению с вертикальной (с 70,8 до 2007,9 мД), что указывает на то, что трещина ГРП распространилась в большей мере в вертикальном направлении и тем самым соединила и увеличила трещины (каналы), связывающие водонасыщенную и нефтяную зоны. И только последующее проведение ремонтно-изоляционных работ позволило снизить обводненность добываемой продукции с 99 до 4-5\%.

Данного негативного последствия вполне можно было избежать, если бы перед проведением работ на скважине данное мероприятие было смоделировано на ГГДМ, которая учитывает особенности геологического строения данной залежи. Одним из основных параметров, на который необходимо обращать внимание при подборе скважин для проведения ГРП, является показатель анизотропии проницаемости пласта.

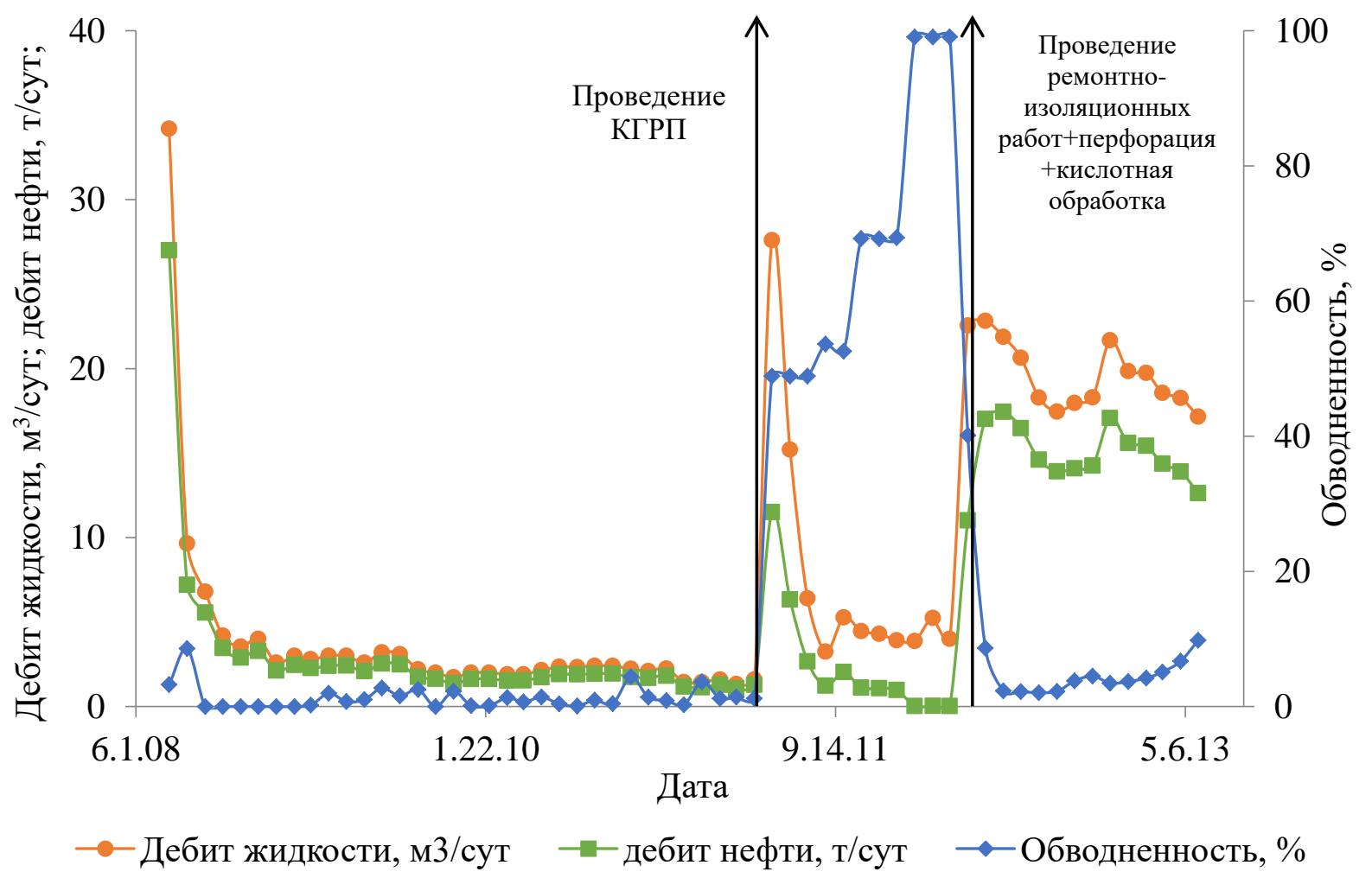

Pис. 1. Эксплуатация скважины № 412 Гагаринского месторождения (Фм залежь) до и после проведения кислотного ГРП

Fig. 1. Operation of well no. 412 of the Gagarinsky field (FM reservoir) before and after acid fracturing 
Таблица 1. Технологические показатели эксплуатации скважины 412 Гагаринского месторождения до и после проведения кислотного ГРП

Table 1. Technological indicators of well operation 412 of the Gagarinsky field before and after acid fracturing

\begin{tabular}{|c|c|c|}
\hline \multirow[b]{2}{*}{$\begin{array}{l}\text { Показатель } \\
\text { Indicator }\end{array}$} & \multicolumn{2}{|c|}{$\begin{array}{l}\text { Дата проведения КГРП - 09.05.2011 } \\
\text { Date of acid fracturing }-09.05 .2011 \\
\end{array}$} \\
\hline & $\begin{array}{c}\text { ГДИ до КГРП - } 08.02 .2011 \\
\text { Hydrodynamic studies before acid fracturing - } \\
08.07 .2011\end{array}$ & $\begin{array}{c}\text { ГДИ после КГРП - 13.05.2011 } \\
\text { Hydrodynamic studies after acid fracturing - } \\
13.05 .2011 \\
\end{array}$ \\
\hline $\begin{array}{l}\text { Пластовое давление, МПа } \\
\text { Reservoir pressure, MPa } \\
\end{array}$ & 6,7 & 6,9 \\
\hline $\begin{array}{l}\text { Забойное давление, МПа } \\
\text { Bottom pressure, MPa }\end{array}$ & 3,7 & 4,4 \\
\hline $\begin{array}{l}\text { Толщина пласта, м } \\
\text { Reservoir thickness, m }\end{array}$ & 8,5 & 8,5 \\
\hline $\begin{array}{l}\text { Кпрод, } \text { м }^{3} /(\text { сут* МПа) } \\
\text { Productivity coefficient, } \mathrm{m}^{3} /(\text { day·MPa) }\end{array}$ & 0,45 & 3,33 \\
\hline $\begin{array}{l}\text { Обводненность, \% } \\
\text { Watering, \% }\end{array}$ & 0,25 & 48,8 \\
\hline $\begin{array}{l}\text { Вертикальная проницаемость, мД } \\
\text { Vertical permeability, md }\end{array}$ & 70,8 & 2007,9 \\
\hline $\begin{array}{l}\text { Горизонтальная проницаемость, мД } \\
\text { Horizontal permeability, md }\end{array}$ & 10,1 & 55,7 \\
\hline
\end{tabular}

\section{Создание ГГДМ, учитывающей геологические} особенности строения карбонатного коллектора

В работе [29] говорится о возможности качественной оценки вертикальной проницаемости карбонатных коллекторов и использования ее в ГГДМ. Авторами статьи была определена анизотропия проницаемости и получены зависимости ее динамики от изменения забойного и пластового давлений. С учетом полученных зависимостей и особенностей геологического строения модифицирована ГГДМ карбонатная фаменская залежь Гагаринского месторождения. Внешний вид модифицированной геолого-гидродинамической модели фаменской залежи Гагаринского месторождения представлен на рис. 2 на примере куба измененных регионов (т. е. регионы фациальных зон с индивидуальными зависимостями множителей проницаемости от пластового давления, красным выделена зона без задания множителей, то есть там проницаемость нединамична).

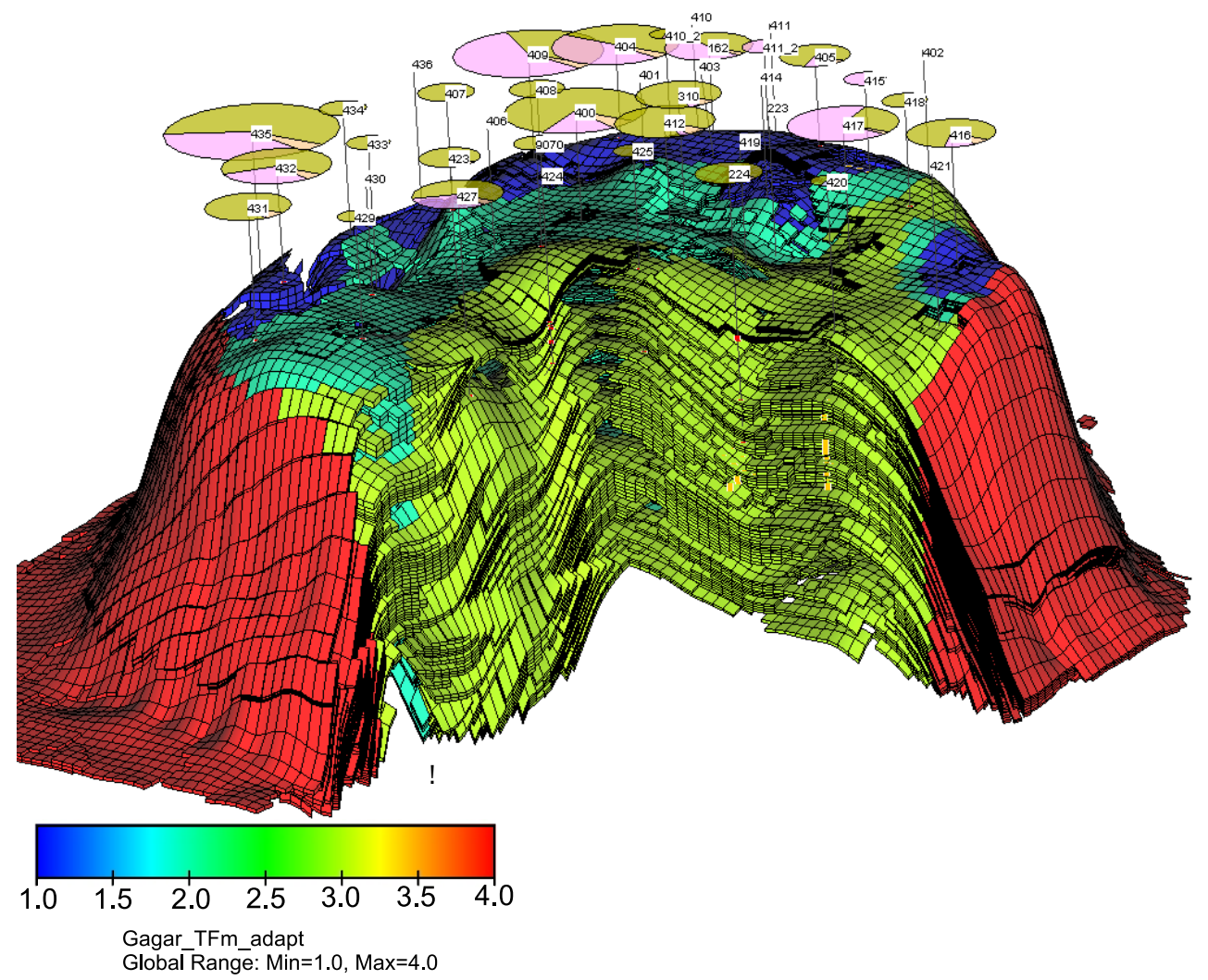

Горизонтально ориентированные овалы - это текущие отборы скважин с указанием в центре номера скважины

Pис. 2. Внешний вид модифицированной ГГДМ фаменской залежи Гагаринского месторождения

Fig. 2. Appearance of a modified geological and hydrodynamic model of the Famennian deposit of the Gagarinsky field 


\section{Прогнозные расчеты технологической} эффективности ГРП

С целью сравнения модифицированной ГГДМ со стандартной (без учета анизотропии проницаемости и наличия естественной трещиноватости и ее динамики в процессе разработки) были проведены расчеты ше- сти ГРП на скважинах, утвержденных в качестве кандидатов для проведения ГРП в соответствии с актуальным планом ГТМ (предложения по проведению геолого-технических мероприятий на 2020-2022 гг. по месторождениям ООО «ЛУКОЙЛ-ПЕРМЬ») (рис. 3).

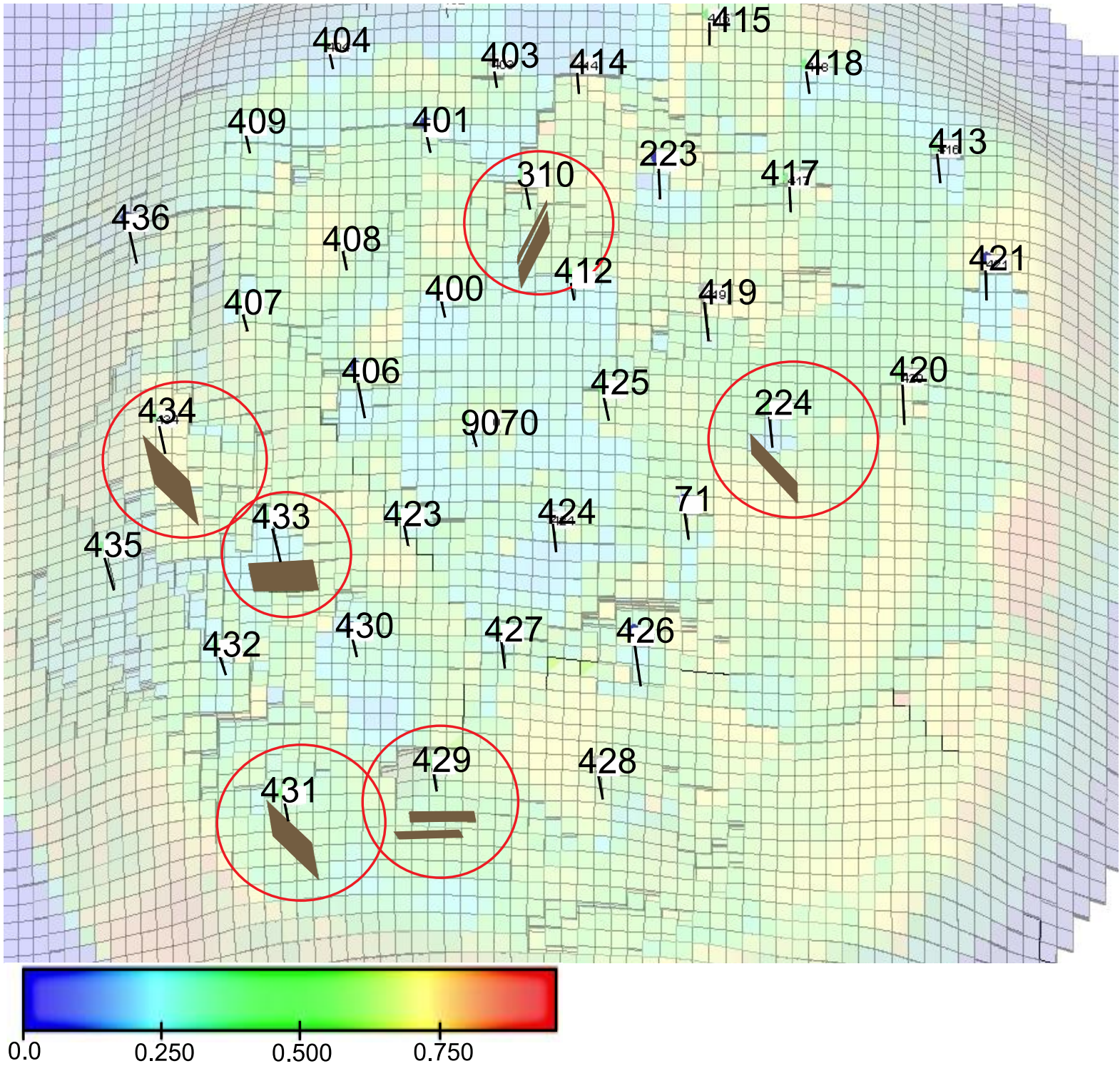

Gagr_TFm: Oil Phase saturation (frac)
Global Range: Min $=0.000$, Max $=0.959$

Нефтенасыщенность

Рис. 3. Расположение скважин-кандидатов для проведения гидравлического разрыва пласта

Fig. 3. Location of candidate wells for hydraulic fracturing

Параметры трещины ГРП (полудлина, проводимость) задавались как средние по объекту по фактически проведенным мероприятиям, их значения составляют 100 м и 1000 мД*м соответственно. Запланированный прирост дебита нефти по каждой из шести скважин в соответствии с программой составляет 6 т/сут. Расположение скважины 434 с визуализированной трещиной ГРП представлено на рис. 4. Моделирование ГРП проводилось с помощью ключевого слова VFRA, которое появилось в симуляторе Tempest с версии 8.0. Особенностями нового подхода к моделированию ГРП является то, что полностью поменялась визуальная составляющая трещины и возможность проведения трещины через неколлектор, что позволяет воспроизводить прорыв через тонкую перемычку.

Расчеты на стандартной ГГДМ (базовый вариант (БАЗ)) показали оптимистичные результаты, суммар- 
ная дополнительная накопленная добыча нефти за время действия эффекта по залежи оценивается в 104,7 тыс. т нефти, таким образом на одну скважину приходится в среднем 17,5 тыс. т. Результаты расчета эффективности ГРП на стандартной ГГДМ представлены на рис. 5.

Гидродинамическая модель, использующая полученные и внедренные зависимости по коэффициенту анизотропии проницаемости, ожидаемо показала рас- четы несколько хуже, суммарная дополнительная накопленная добыча нефти за время действия эффекта по залежи оценивается в 25,4 тыс. т нефти, таким образом на одну скважину приходится в среднем 4,2 тыс. т. Результаты расчета эффективности ГРП на модифицированной ГГДМ показаны на рис. 6.

Сравнение результатов технологической эффективности ГРП на скважинах-кандидатах представлены в табл. 2.

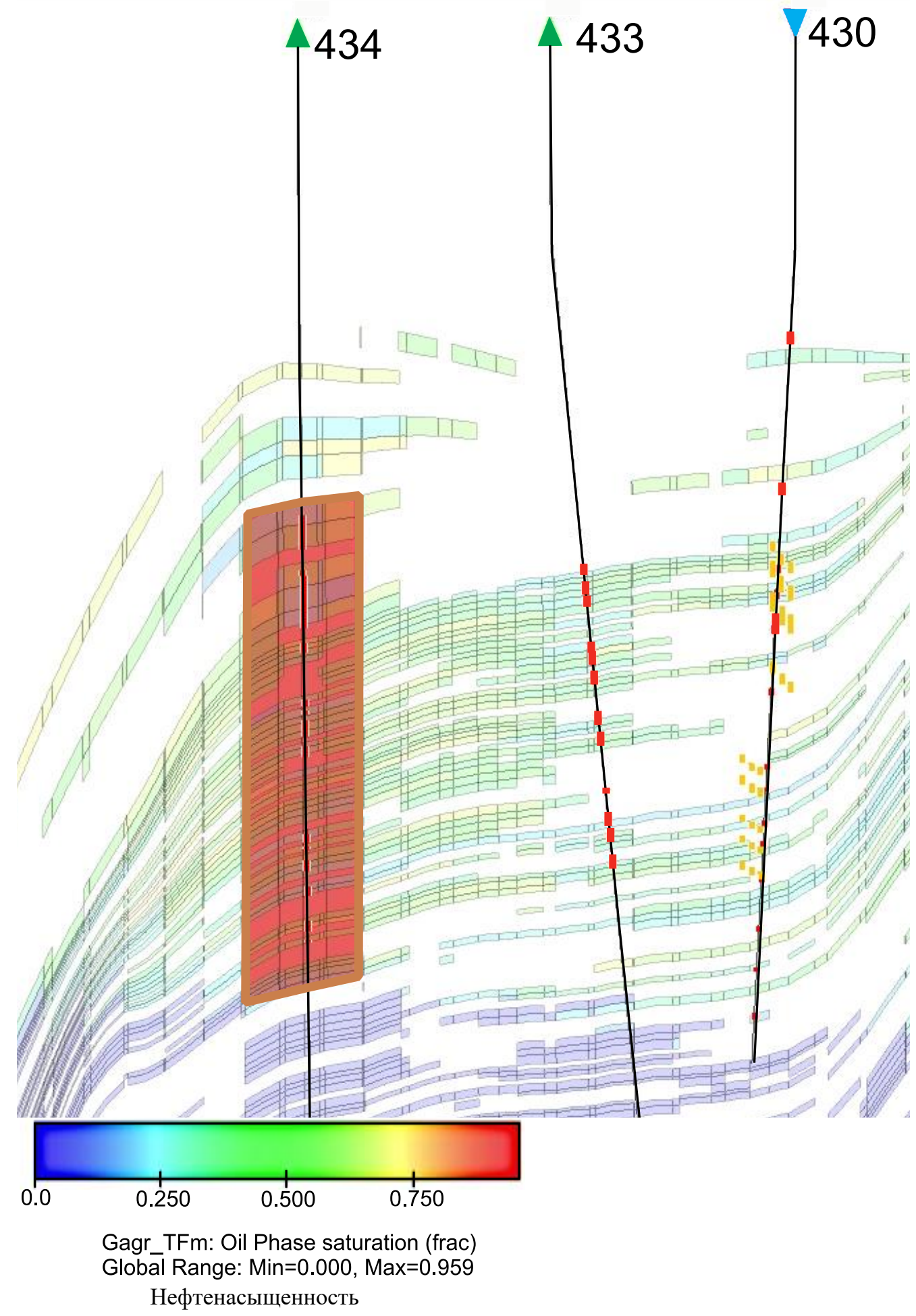

Pис. 4. Визуализация трещзины гидравлического разрыва пласта на скважсине-кандидате № 434

Fig. 4. Visualization of hydraulic fracture at candidate well 434 

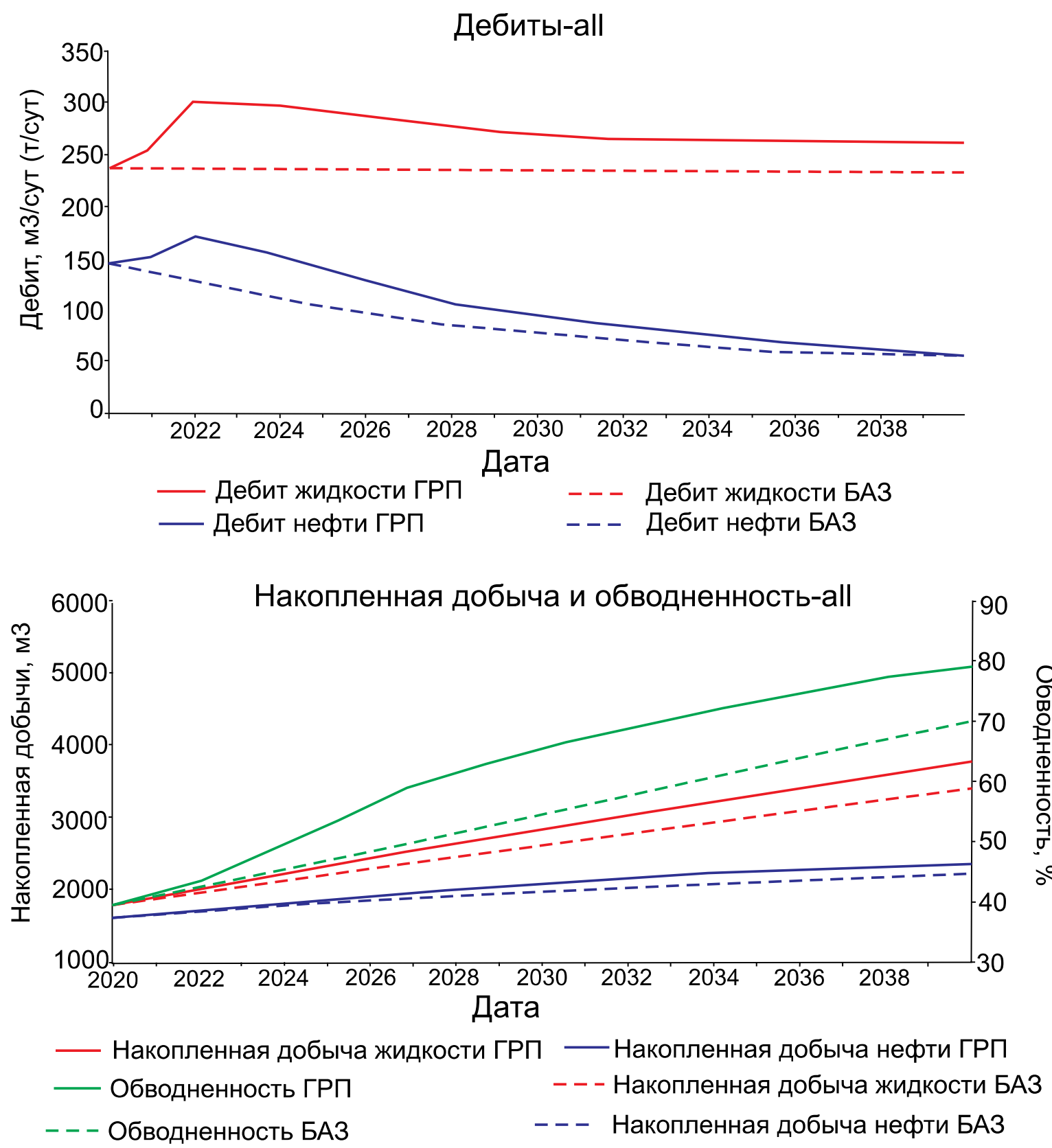

Pис. 5. Результаты расчета гидравлического разрыва пласта на стандартной ГГДМ

Fig. 5. Results of calculation of hydraulic fracturing on a standard geological and hydrodynamic model

Таблица 2. Сравнение результатов эффективности гидравлического разрыва пласта, полученные на стандартной и модифицированной геолого-гидродинамической моделях

Table 2. Comparison of results of hydraulic fracturing efficiency obtained on standard and modified geological and hydrodynamic models

\begin{tabular}{|c|c|c|c|c|c|c|c|c|c|c|c|c|}
\hline \multirow{2}{*}{$\begin{array}{l}\text { Параметр } \\
\text { Parameter }\end{array}$} & \multicolumn{12}{|c|}{ Скважина/Well } \\
\hline & \multicolumn{2}{|c|}{224} & \multicolumn{2}{|c|}{310} & \multicolumn{2}{|c|}{429} & \multicolumn{2}{|c|}{431} & \multicolumn{2}{|c|}{433} & \multicolumn{2}{|c|}{434} \\
\hline $\begin{array}{l}\text { Год проведения ГРП } \\
\text { Year of hydraulic fracturing }\end{array}$ & \multicolumn{2}{|c|}{2021} & \multicolumn{2}{|c|}{2021} & \multicolumn{2}{|c|}{2020} & \multicolumn{2}{|c|}{2021} & \multicolumn{2}{|c|}{2020} & \multicolumn{2}{|c|}{2021} \\
\hline $\begin{array}{l}\text { Мгновенный прирост дебита нефти, т/сут } \\
\text { Instant increase in oil production rate, t/day }\end{array}$ & $6,0 *$ & $3,1 * *$ & 4,9 & $-1,8$ & 5,7 & 1,5 & 6,2 & 6,2 & 5,9 & 5,0 & 5,7 & 4,9 \\
\hline $\begin{array}{l}\text { Мгновенный прирост обводненности, \% } \\
\text { Instant increase in watering,\% }\end{array}$ & 19,3 & 27,7 & 32,2 & 37,2 & 24,2 & 30,4 & 0,7 & 0,7 & 6,9 & 18,4 & $-5,3$ & 17,7 \\
\hline
\end{tabular}

Примечание: слева показан результат на стандартной модели (*), справа - на модифицированной (**).

Note: result on the standard model is on the left (*), result on the modified one is on the right (**). 


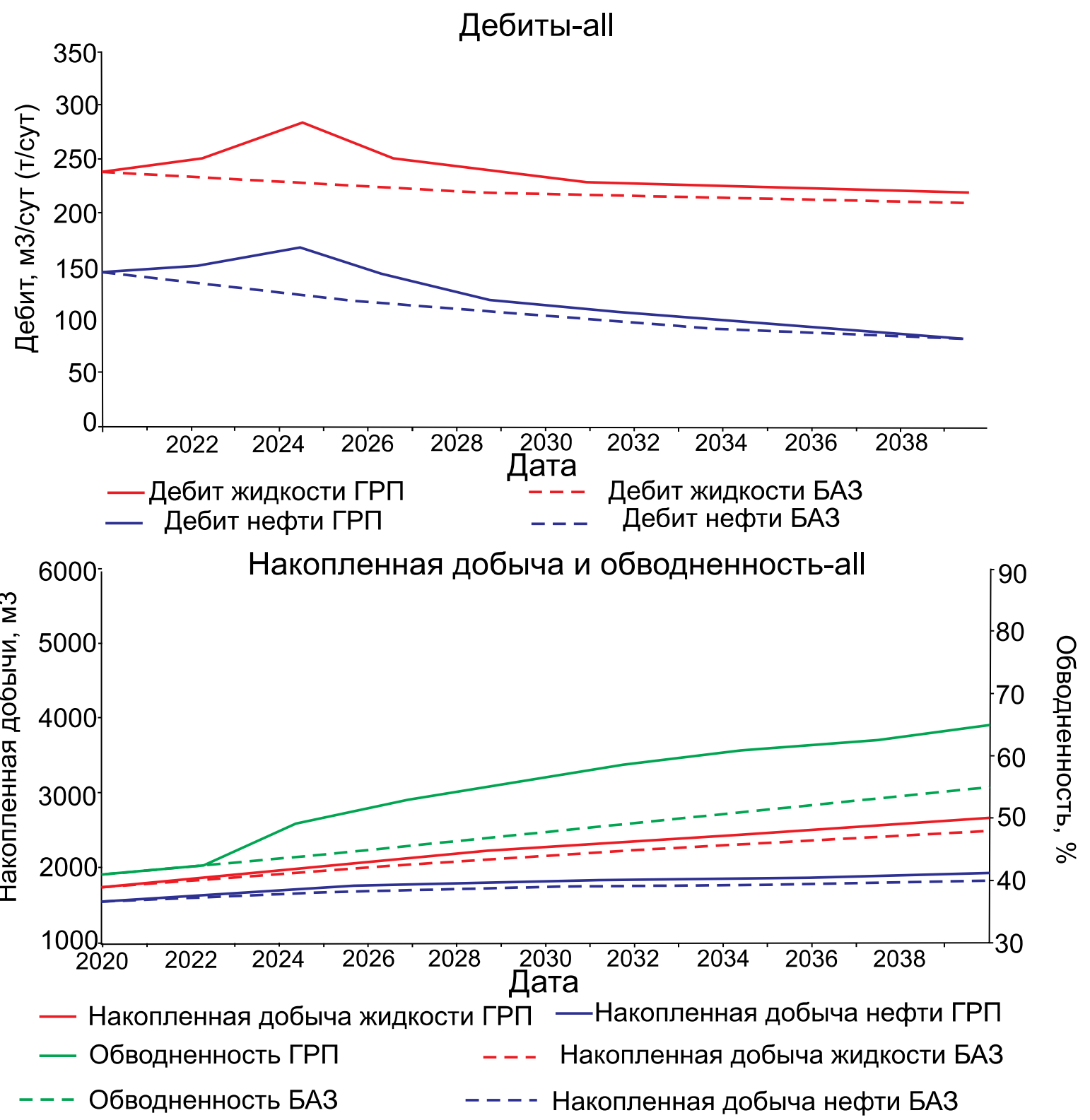

Pис. 6. Результаты расчета гидравлического разрыва пласта на модифицированной ГГДМ

Fig. 6. Results of calculation of hydraulic fracturing on a modified geological and hydrodynamic model

Таким образом, единственной рекомендуемой скважиной-кандидатом является скважина № 431, которая имеет одинаковый мгновенный прирост дебита нефти и обводненности, однако эффект закончился в три раза быстрее, и суммарная накопленная дополнительная добыча нефти от ГРП составила 7 тыс. т. Из табл. 2 видно, что при прогнозных расчетах ГРП на стандартной ГГДМ без учета особенностей геологического строения карбонатных массивных залежей существует риск недостижения плановых приростов дебитов, по некоторым скважинам значительно возрастает обводненность добываемой продукции и, как следствие, все это может привести к отрицательному экономическому эффекту.

Дополнительная добыча нефти, рассчитанная в ГГДМ (стандартная (базовая) и модифицированная) по результатам проведения ГРП на утвержденных скважинах-кандидатах, представлена на рис. 7.

\section{Оценка экономической эффективности запланированных ГРП}

По результатам моделирования ГРП на шести скважинах-кандидатах с использованием стандартной и модифицированной ГГДМ рассчитана экономическая составляющая этих мероприятий, представленная в табл. 3. Также на рис. 8 представлен график, отражающий чувствительность мероприятий к тем или иным изменениям. Данный тип графика позволяет визуально оценивать риски и определить критические точки планируемых мероприятий.

Анализируя полученные результаты расчета, стоить выделить основные моменты: ЧДД и индекс доходности, рассчитанные на модифицированной ГГДМ оказались в 3,7 и 2,8 раз меньше по сравнению со стандартной, что является, несомненно, важным показателем при подборе скважин-кандидатов для 
планирования ГТМ. В целом стоить отметить, что предлагаемые ГРП на скважинах являются экономически эффективными, но со значительной разницей в экономических показателях между моделями. Наиболее чувствительным параметром является цена нефти, при снижении цены более чем на 26 \% проект становится нерентабельным (рис. 8).
Таким образом, расчеты на модифицированной модели показывают, что, вероятнее всего, экономический эффект будет не таким оптимистичным, как при стандартной модели, так как учитываются риски при реализации технологии ГРП. Соответственно с помощью модифицированной цифровой модели можно более обоснованно подбирать скважины-кандидаты под проведения ГРП

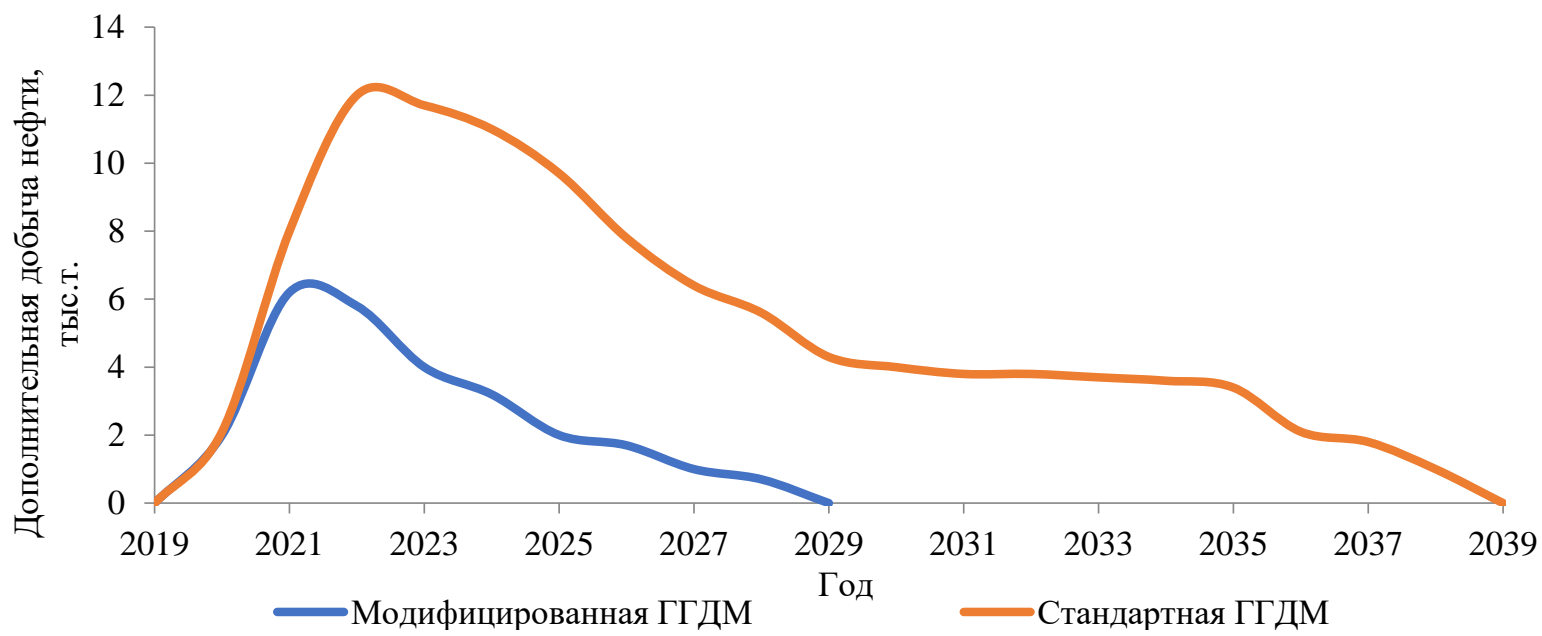

Pис. 7. Дополнительная добыча нефти в динамике, рассчитанная в геолого-гидродинамической модели (стандартная и модифицированная) по результатам проведения гидравлического разрыва пласта на утвержденных скважинах-кандидатах

Fig. 7. Additional oil production in dynamics, calculated in a geological and hydrodynamic model (standard and modified) based on the results of hydraulic fracturing at approved candidate wells

Таблица 3. Экономические показатели планируемых мероприятий по гидравлическому разрыву пласта

Table 3. Economic indicators of the planned hydraulic fracturing

\begin{tabular}{|l|c|c|}
\hline \multicolumn{1}{|c|}{$\begin{array}{c}\text { Показатели экономической эффективности } \\
\text { Indicators of есоnоmic efficiency }\end{array}$} & $\begin{array}{c}\text { Стандартная ГГДМ } \\
\text { Standard geological and hydrodynamic } \\
\text { model }\end{array}$ & $\begin{array}{c}\text { Модифицированная ГГДМ } \\
\text { Modified geological and hydrodynamic } \\
\text { model }\end{array}$ \\
\hline $\begin{array}{l}\text { Чистый дисконтированный доход (ЧДД), млн p. } \\
\text { Net present value (NPV), million rubles }\end{array}$ & 364,59 & 97,60 \\
\hline $\begin{array}{l}\text { Индекс доходности } \\
\text { Yield index }\end{array}$ & 8,18 & 2,92 \\
\hline $\begin{array}{l}\text { Срок окупаемости, мес. } \\
\text { Payback period, months }\end{array}$ & 6 & 10 \\
\hline $\begin{array}{l}\text { Hopма доходности } \\
\text { Rate of return }\end{array}$ & 2,16 & 1,25 \\
\hline
\end{tabular}

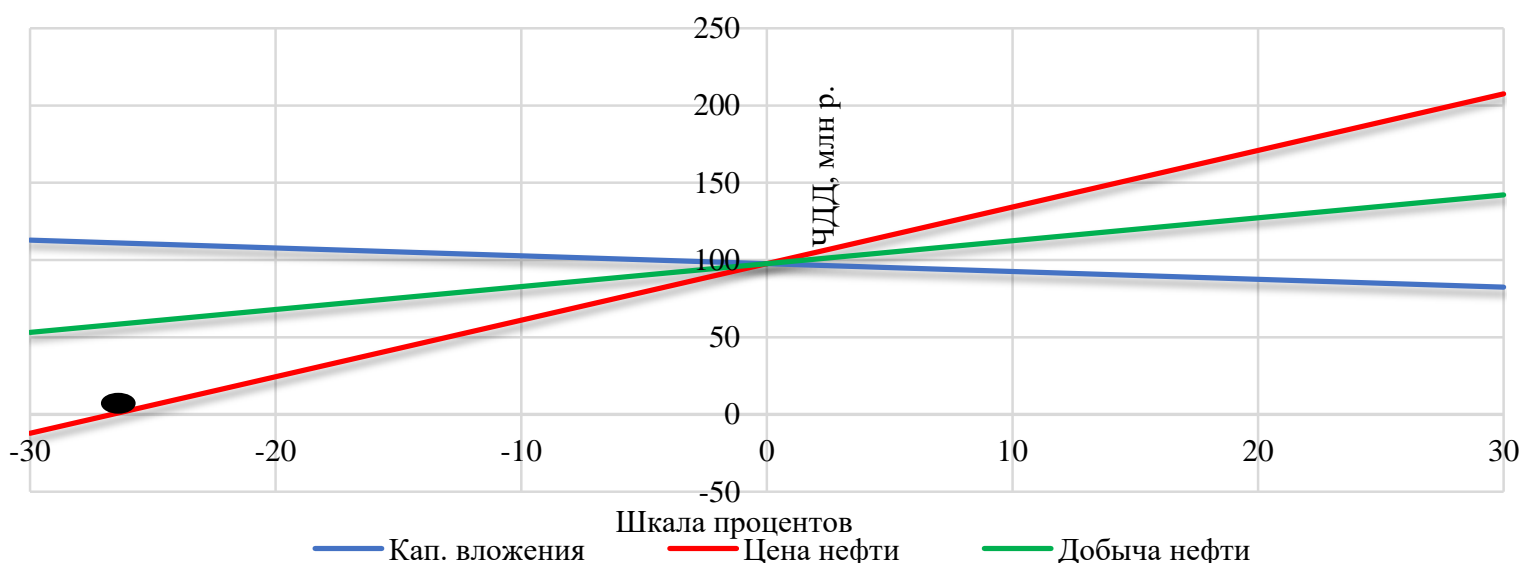

Pис. 8. Диаграмма результатов анализа чувствительности, полученная по результатам расчета в модифицированной ГГДМ

Fig. 8. Diagram of sensitivity analysis results, obtained from the calculation results in a modified geological and hydrodynamic model 


\section{Заключение}

Полученные в ходе моделирования технологии ГРП на шести скважинах-кандидатах результаты показали, что использование стандартной (применяется на сегодняшний момент) по сравнению с модифицированной ГГДМ (учитывает анизотропию проницаемости и трещиноватость) приводит к завышению одних технологических показателей (прирост дебита нефти и накопленная добыча нефти) и к занижению других (обводненность добываемой продукции), что соответственно в последующем может привести к незначительной или отрицательной экономической составляющей мероприятий.

\section{СПИСОК ЛИТЕРАТУРЫ}

1. Henderson N., Pena L. Simulating effects of the permeability anisotropy on formation of viscous fingers during waterflood operations // Journal of petroleum science and engineering. 2017. - V. 153. - P. 178-186.

2. Jackson R.R., Banerjee R. Application of reservoir simulation and history matching methods to MDT vertical interference testing and determination of permeability anisotropy // 8th European Conference on the mathematics of oil recovery. Freiberg, Germany, 3-6 September 2002.

3. Мухаметшин В.В., Андреев В.Е. Повышение эффективности оценки результативности технологий, направленных на расширение использования ресурсной базы месторождений с трудноизвлекаемыми запасами // Известия Томского политехнического университета. Инжиниринг георесурсов. - 2018. T. 329. - № 8. - C. 30-36

4. Причины увеличения обводненности в скважинах после проведения гидравлического разрыва в неоднородных пластах / А.С. Валеев, М.Р. Дулкарнаев, Ю.А. Котенев, Ш.Х. Султанов, Л.С. Бриллиант // Известия Томского политехнического университета. Инжиниринг георесурсов. - 2018. - Т. 329. - № 6. C. $140-147$.

5. Modeling fracture propagation and cleanup for dry nanoparticlestabilized-foam fracturing fluids / A. Qajar, Z. Xue, A.J. Worthen, K.P. Johnston, M. Prodanovic // Journal of Petroleum Science and Engineering. - October 2016. - V. 146. - P. 210-221.

6. On the use of high aspect ratio finite elements to model hydraulic fracturing in deformable porous media / O.L. Manzoli, P.R. Cleto M. Sanchez, L.J.N. Guimaraes, M.A. Maedo // Computer Methods in Applied Mechanics and Engineering. - June 2019. - V. 350. P. 57-80.

7. Impact of the distance between pre-existing fracture and wellbore on hydraulic fracture propagation / Bo Zhang, Jiyang Liu, S.G. Wang, Shucai Li, Weimin Yang // Journal of Natural Gas Science and Engineering. - September 2018. - V. 57. - P. 155-165.

8. Восстановление и повышение продуктивности добывающих скважин каширского и подольского объектов на одном из нефтяных месторождений Пермского края / А.С. Вотинов, С.А. Дроздов, В.Л. Малышева, В.А. Мордвинов // Вестник Пермского национального исследовательского политехнического университета. Геология. Нефтегазовое и горное дело. 2018. - T. 18. - № 2. - C. 140-148.

9. Экономидес М., Олини Р., Валько П. Унифицированный дизайн гидроразрыва пласта: от теории к практике. - М.; Ижевск: Институт компьютерных технологий, 2007. -237 с.

10. Развитие технологий гидравлического разрыва пласта на месторождениях Пермского края / В.Л. Воеводкин, А.А. Алероев, Т.Р. Балдина, А.В. Распопов, А.С. Казанцев, С.А. Кондратьев // Нефтяное хозяйство. - 2018. - № 11. - С. 108-113.

11. Черепанов С.С., Чумаков Г.Н., Пономарева И.Н. Результаты проведения кислотного гидроразрыва пласта с проппантом на турнейско-фаменской залежи Озерного месторождения // Вестник Пермского национального исследовательского политехнического университета. Геология. Нефтегазовое и горное дело. - 2015. - Т. 14. - № 16. - С. 70-76.
В виду того что в нефтяных компаниях вводятся все больше объектов с трудноизвлекаемыми и нетрадиционными запасами углеводородного сырья перед компаниями ставятся все новые актуальные задачи, заключающиеся в подборе и развитии новых технологий. В этой связи предопределяются направления развития технологий геолого-гидродинамического моделирования: акцент делается на более полные и совершенные модели, описывающие сложные процессы, происходящие в пласте. Предлагаемые подходы и приемы, описанные в статье, показали свою эффективность и могут быть использованы для дальнейшего расширения на другие карбонатные объекты.

12. Галкин В.И., Пономарева И.Н., Колтырин А.Н. Разработка вероятностно-статистическими моделей для оценки эффективности применения проппантного гидравлического разрыва пласта (на примере объекта Тл-Бб Батырбайского месторождения) // Вестник Пермского национального исследовательского политехнического университета. Геология. Нефтегазовое и горное дело. - 2018. - Т. 17. - № 1. - С. 37-49.

13. Бархатов Э.А., Яркеева Н.Р. Эффективность применения многозонного гидроразрыва пласта в горизонтальных скважинах // Известия Томского политехнического университета. Инжиниринг георесурсов. - 2017. - Т. 328. - № 10. - С. 50-58.

14. Проведение проппантных гидроразрывов низкопроницаемых пластов на нефтяных месторождениях Немецкого автономного округа / А.А. Алероев, С.А. Кондратьев, Р.Р. Шарафаев, Д.В. Новокрещенных, В.А. Жигалов // Нефтяное хозяйство. 2017. - № 9. - C. 108-111.

15. Мухаметшин В.В. Обоснование трендов повышения степени выработки запасов нефти нижнемеловых отложений Западной Сибири на основе идентификации объектов // Известия Томского политехнического университета. Инжиниринг георесурсов. -2018 . - T. 329. - № 5. - С. 117-124.

16. Морозов П.Е. Псевдоскин-фактор и оптимальная проводимость трещины гидроразрыва в круговом пласте // Нефтяное хозяйство. - 2019. - № 3. - С. 74-77.

17. Boahua Liu, Yan Jin, Mian Chen. Influence of vugs in fracturedvuggy carbonate reservoirs on hydraulic fracture propagation based on laboratory experiments // Journal of Structural Geology. - July 2019. - V. 124. - P. 143-150.

18. HanYi Wang, Sharma M.M. Modeling of hydraulic fracture closure on proppants with proppant settling // Journal of Petroleum Science and Engineering. - December 2018. - V. 171. - P. 636-645.

19. Modeling fate and transport of hydraulic fracturing fluid in the presence of abandoned wells / R. Taherdangkoo, A. Tatomir, T. Anighoro, M. Sauter // Journal of Contaminant Hydrology. February 2019. - V. 221. - P. 58-68.

20. Garagash I.A., Osiptsov A.A., Boronin S.A. Dynamic dridging of proppant particles in a hydraulic fracture // International Journal of Engineering Science. - February 2019. - V. 135. - P. 86-101.

21. Livio Santos, Arash Dahi Taleghani, Guoqiang Li. Expandable proppants to moderate production drop in hydraulically fractured wells // Journal of Natural Gas Science and Engineering. - July 2018. - V. 55. - P. 182-190.

22. Computer simulation of hydraulic fractures / J. Adachi, E. Siebrits, A. Peirce, J. Desroches // International Journal of Rock Mechanics and Mining Science. - July 2007. - V. 44. - Iss. 5. - P. 739-757.

23. HanYi Wang. Hydraulic fracture propagation in naturally fractured reservoir: complex fracture of fracture networks // Journal of Natural Gas Science and Engineering. URL: https://www.sciencedirect.com/science/article/pii/S187551001930 1556 (дата обращения 15.04.2020).

24. Hydro-mechanical modeling of hydraulic fracture propagation and its interactions with frictional natural fractures / J.A.R. Cordero, E.M. Sanchez, D. Roehl, L.C. Pereira // Computers and Geotechnics. - July 2019. - V. 111. - P. 290-300.

25. Yaghoubi A. Hydraulic fracturing modeling using a discrete fracture network in the Barnett Shale // International Journal of 
Rock Mechanics and Mining Science. - July 2019. - V. 119. P. 98-108.

26. An experimental investigation into the characteristics of hydraulic fracturing and fracture permeability after hydraulic fracturing in granite / Yanjun Zhang, Yueqiang Ma, Zhongjun Hu, Honglei Lei, Qian Zhang // Renewable Energy. - September 2019. - V. 140. P. 615-624.

27. Patel S.M., Sondergeld C.H., Rai C.S. Hydraulic fracture permeability estimation using stimulation pressure data // International Journal of Rock Mechanics and Mining Science. January 2018. - V. 101. - P. 50-53.

\section{Информация об авторах}

Менгалиев А.Г., инженер 2-й категории отдела создания и мониторинга гидродинамических моделей приоритетных месторождений филиала ООО «ЛУКОЙЛ-Инжиниринг» «ПермНИПИнефть» в г. Перми.

Мартюшев Д.А., кандидат технических наук, доцент кафедры Нефтегазовые технологии Пермского национального исследовательского политехнического университета.
28. Zhang Simulation of proppant distribution in hydraulically fractured shale network during shut-in period / Fei Wang, Baoman Li, Qiaoyn Chen, Shicheng // Journal of Petroleum Science and Engineering. - July 2019. - V. 178. - P. 467-474.

29. Менгалиев А.Г., Мартюшев Д.А. Определение и изменение показателя анизотропии проницаемости в процессе разработки карбонатной залежи нефтяного месторождения // Инженернефтяник. - 2019. - № 1 (46). - С. 27-33.

Поступила 03.06.2020 г. 


\title{
ASSESSMENT OF TECHNOLOGICAL AND ECONOMIC EFFICIENCY OF FRACTURING FORMATIONS USING GEOLOGICAL AND HYDRODYNAMIC MODELS, CONSIDERING FEATURES OF CARBONATE RESERVOIR STRUCTURE
}

\author{
Alexander G. Mengaliev1, \\ mengaliev.alexandr@gmail.com \\ Dmitriy A. Martyushev², \\ martyushevd@inbox.ru \\ 1 Branch of LLC «LUKOIL-Engineering» «PermNIPIneft» in Perm, \\ 29, Sovetskoy Armii street, Perm, 614000, Russia. \\ 2 Perm National Research Polytechnic University, \\ 29, Komsomolskiy avenue, Perm, 614990, Russia.
}

The relevance of the discussed issue. Currently, one of the most effective methods to intensify oil production in the Perm Territory and throughout the world is hydraulic fracturing. The conducted hydraulic fracturing measures are often accompanied by an increase in water cut of the produced products due to the fact that during the event the hydraulic fracturing spread in vertical direction and thereby increased the channels connecting the water-saturated and oil zones. This negative effect could be avoided if before the work on the well the event was modeled on a geological and hydrodynamic model that takes into account the specific geological structure of this deposit. Knowledge of the structural features of carbonate deposits allows us to significantly clarify and improve the existing geological and hydrodynamic models. Accordingly, the use of geological and hydrodynamic models that take into account the structural features of carbonate objects will allow choosing a rational system of development and ensuring optimal geological and technological measures and, as a result, will lead to a decrease in economic risks.

The main aim of the study is to evaluate the technological and economic efficiency of hydraulic fracturing using a hydrodynamic model that takes into account the geological features of the carbonate reservoir structure.

Object: Famennian carbonate reservoir of the Gagarinsky field.

Methods: processing of geological research data; use of the Tempest hydrodynamic simulator version 8.3.1 from Roxar.

The results obtained during the simulation of hydraulic fracturing at six candidate wells showed that the use of the standard model (currently used) compared to the modified geological and hydrodynamic model (takes into account the permeability anisotropy and fracturing) leads to overestimation of some technological indicators (increase in oil production and cumulative oil production) and to underestimation of others (the water content of the extracted products), which subsequently can lead to a distortion in economic component of events. The economic analysis made it possible to establish that the net discounted income calculated on the modified geological and hydrodynamic model turned out to be 3,7 times less than the standard one, which is undoubtedly the important indicators in selecting candidates for planning geological and technological measures.

\section{Key words:}

Vertical and horizontal permeability, fracturing, increase in water cut, increase in oil production rate, economic feasibility, additional oil production.

\section{REFERENCES}

1. Henderson N., Pena L. Simulating effects of the permeability anisotropy on formation of viscous fingers during waterflood operations. Journal of petroleum science and engineering, 2017, vol. 153, pp. $178-186$

2. Jackson R.R., Banerjee R. Application of reservoir simulation and history matching methods to MDT vertical interference testing and determination of permeability anisotropy. $8^{\text {th }}$ European Conference on the mathematics of oil recovery. Freiberg, Germany, 3-6 September, 2002.

3. Mukhametshin V.V., Andreev V.E. Improving the efficiency of assessing the effectiveness of technologies aimed at expanding the use of the resource base of deposits with hard-to-recover reserves. Bulletin of the Tomsk Polytechnic University. Geo Assets engineering, 2018, vol. 329, no. 8, pp. 30-36. In Rus.

4. Valeev A.S., Dulkarnaev M.R., Kotenev Yu.A., Sultanov Sh.H., Brilliant L.S. The reasons for the increase in water cut in wells after the hydraulic fracturing in heterogeneous reservoirs. Bulletin of the Tomsk Polytechnic University. Geo Assets engineering, 2018, vol. 329, no. 6, pp. 140-147. In Rus.

5. Qajar A., Xue Z., Worthen A.J., Johnston K.P., Prodanovic M. Modeling fracture propagation and cleanup for dry nanoparticlestabilized-foam fracturing fluids. Journal of Petroleum Science and Engineering, October 2016, vol.146, pp. 210-221.
6. Manzoli O.L., Cleto P.R., Sanchez M., Guimaraes L.J.N., Maedo M.A. On the use of high aspect ratio finite elements to model hydraulic fracturing in deformable porous media. Computer Methods in Applied Mechanics and Engineering, June 2019, vol. 350 , pp. $57-80$.

7. Bo Zhang, Jiyang Liu, Wang S.G., Shucai Li, Weimin Yang. Impact of the distance between pre-existing fracture and wellbore on hydraulic fracture propagation. Journal of Natural Gas Science and Engineering, September 2018, vol. 57, pp. 155-165.

8. Votinov A.S., Drozdov S.A., Malysheva V.L., Mordvinov V.A. Restoration and increase of productivity of producing wells of Kashirsky and Podolsk facilities at one of the oil fields of the Perm Territory. Bulletin of the Perm National Research Polytechnic University. Geology. Oil and gas and mining, 2018, vol. 18, no. 2, pp. 140-148. In Rus.

9. Economides M., Olini R., Valko P. Unificirovanny dizayn gidrorazryva plasta: ot teorii $k$ praktike [Unified reservoir fracture design: from theory to practice]. Moscow, Izhevsk, Institute of Computer Technologies Publ., 2007. 237 p.

10. Voevodkin V.L., Aleroev A.A., Baldina T.R., Raspopov A.V., Kazantsev A.S., Kondratiev S.A. Development of hydraulic fracturing technologies in the fields of the Perm Krai. Oil industry, 2018, no. 11, pp. 108-113. In Rus.

11. Cherepanov S.S., Chumakov G.N., Ponomareva I.N. Results of acid fracturing with proppant at the Tournaisian-Famennian 
deposit of the Ozernoye field. Perm National Research Polytechnic University. Geology. Oil and gas and mining, 2015, vol. 14, no. 16, pp. 70-76. In Rus.

12. Galkin V.I., Ponomareva I.N., Koltyrin A.N. Development of probabilistic-statistical models for evaluating the effectiveness of the use of proppant hydraulic fracturing (on the example of the T$\mathrm{Bb}$ facility of the Batyrbai field). Perm National Research Polytechnic University. Geology. Oil and gas and mining, 2018, vol. 17, no. 1, pp. 37-49. In Rus.

13. Barkhatov E.A., Yarkeeva N.R. The effectiveness of multi-zone hydraulic fracturing in horizontal wells. Bulletin of the Tomsk Polytechnic University. Geo Assets engineering, 2017, vol. 328, no. 10, pp 50-58. In Rus.

14. Aleroev A.A., Kondratiev S.A., Sharafayev R.R., Novokreshchennykh D.V., Zhigalov V.A. Carrying proppant fracturing low permeability reservoirs in the oil fields of the German autonomous region. Oil Industry, 2017, no. 9, pp 108-111 In Rus.

15. Mukhametshin V.V. Justification of trends in increasing the degree of oil reserves in the Lower Cretaceous deposits of Western Siberia based on the identification of objects. Bulletin of the Tomsk Polytechnic University. Geo Assets engineering, 2018, vol. 329, no. 5, pp. 117-124. In Rus.

16. Morozov P.E. Pseudoskin factor and optimum fracture conductivity of a fracture in a circular reservoir. Oil industry, 2019 no. 3, pp. 74-77. In Rus.

17. Boahua Liu, Yan Jin, Mian Chen. Influence of vugs in fracturedvuggy carbonate reservoirs on hydraulic fracture propagation based on laboratory experiments. Journal of Structural Geology, July 2019 , vol. 124 , pp. 143-150.

18. HanYi Wang, Sharma M.M. Modeling of hydraulic fracture closure on proppants with proppant settling. Journal of Petroleum Science and Engineering, December 2018, vol. 171, pp. 636-645.

19. Taherdangkoo R., Tatomir A., Anighoro T., Sauter M. Modeling fate and transport of hydraulic fracturing fluid in the presence of abandoned wells. Journal of Contaminant Hydrology, February 2019, vol. 221, pp. 58-68.

20. Garagash I.A., Osiptsov A.A., Boronin S.A. Dynamic dridging of proppant particles in a hydraulic fracture. International Journal of Engineering Science, February 2019, vol. 135, pp. 86-101.
21. Livio Santos, Arash Dahi Taleghani, Guoqiang Li. Expandable proppants to moderate production drop in hydraulically fractured wells. Journal of Natural Gas Science and Engineering, July 2018, vol. 55, pp. 182-190.

22. Adachi J., Siebrits E., Peirce A., J. Desroches. Computer simulation of hydraulic fractures. International Journal of Rock Mechanics and Mining Science, July 2007, vol. 44, Iss. 5, pp. 739-757.

23. HanYi Wang. Hydraulic fracture propagation in naturally fractured reservoir: complex fracture of fracture networks. Journal of Natural Gas Science and Engineering. Available at: https://www.sciencedirect.com/science/article/pii/S187551001930 1556 (accessed 15 April 2020).

24. Cordero J.A.R., Sanchez E.M., Roehl D., Pereira L.C. Hydromechanical modeling of hydraulic fracture propagation and its interactions with frictional natural fractures. Computers and Geotechnics, July 2019, vol. 111, pp. 290-300.

25. Ali Yaghoubi. Hydraulic fracturing modeling using a discrete fracture network in the Barnett Shale. International Journal of Rock Mechanics and Mining Science, July 2019, vol. 119, pp. 98-108.

26. Yanjun Zhang, Yueqiang Ma, Zhongjun Hu, Honglei Lei, Qian Zhang. An experimental investigation into the characteristics of hydraulic fracturing and fracture permeability after hydraulic fracturing in granite. Renewable Energy, September 2019, vol. 140 pp. 615-624.

27. Patel S.M., Sondergeld C.H., Rai C.S. Hydraulic fracture permeability estimation using stimulation pressure data. International Journal of Rock Mechanics and Mining Science, January 2018, vol. 101, pp. 50-53.

28. Fei Wang, Baoman Li, Qiaoyn Chen, Shicheng Zhang. Simulation of proppant distribution in hydraulically fractured shale network during shut-in period. Journal of Petroleum Science and Engineering, July 2019, vol. 178, pp. 467-474.

29. Mengaliev A.G., Martyushev D.A. Determination and change of permeability anisotropy in the development of a carbonate reservoir of an oil field. Petroleum engineer, 2019, no. 1 (46), pp. 27-33. In Rus.

Received: 3 June 2020.

Information about the authors

Alexander G. Mengaliev, $2^{\text {nd }}$ category engineer, branch of LLC «LUKOIL-Engineering» «PermNIPIneft» in Perm. Dmitriy A. Martyushev, Cand. Sc., assistant professor, Perm National Research Polytechnic University. 